\title{
A Study of cavitation induced surface erosion in abrasive waterjet cutting systems
}

\author{
J. Madadnia ${ }^{1, *}$, D.K. Shanmugam², T. Nguyen ${ }^{2}$ and J. Wang ${ }^{2}$ \\ ${ }^{1}$ Faculty of Engineering, University of Technology, Sydney, PO.Box 123, Broadway, NSW 2007, Australia. \\ ${ }^{2}$ School of Mechanical and Manufacturing Engineering, The University of New South Wales, NSW 2052, Australia
}

\begin{abstract}
Current jet cutting systems experience severe nozzle erosion and associated maintenance and downtime costs. An experimental investigation was conducted to qualitatively and quantitatively analyse the generation of cavitation in a high pressure water jet cutting system, and to characterise cavitation induced accelerated surface erosion by slurries. The analysis of surface morphology indicates that the shearing induced by cavitation played a major role in the erosion process. The results promise a feasible solution to reduce nozzle wear, and to enhance material removal in the jet cutting process.
\end{abstract}

Keywords: Cavitation erosion; Abrasive erosion; Submerged high pressure jet; Material removal; Erosion wear; Abrasive waterjet; AWJ.

\section{Introduction}

The high pressure components in the abrasive suspension jet (ASJ) system are severely eroded by the pressurised slurries. In an entrainment abrasive waterjet (AWJ) system, only pressurised water is pumped through the system, and dry-abrasives with air are then admitted into the water, leading to wear in the nozzle or mixing tube. It is therefore important to investigate ways to reduce the wear and to improve cutting efficiency in those systems. The entrained air in a high speed slurry jet can significantly generate mist at the nozzle exit. The mist is harmful if soluble chemicals are added to water to improve cutting performance of the jet and the issue of occupational health and safety needs to be considered [1-4].

The erosion accelerated by cavitation has been investigated in many material handling systems such as vortex-amplifiers [5], conical diffusers [6], valves [7], and low speed waterjet nozzles [8]. These studies have shown that at the condition of liquid flow where tensile forces can induce nucleation of vapour bubbles, the collapse of these bubbles at a higher pressure on a surface

\footnotetext{
* Corresponding Author: Fax: +61 29514 2655, E-mail: j.madadnia@uts.edu.au (J. Madadnia).
} 
MADADNIA, J, SHANMUGAM, D.K., NGUYEN, T. and WANG, J., A study of cavitation induced surface erosion in abrasive waterjet cutting systems. 2008 Advanced Materials Research Vols. 53-54 (2008) pp 357362, online at http://www.scientific.net (C) (2008) Trans Tech Publications, Switzerland

result in severe surface erosion. This phenomenon has also been studied in high pressure waterjet cutting devices and a significant improvement in the cutting performance was found by utilizing cavitation, whereby the cutting depths were increased by $100 \%$ and $500 \%$ when cavitation was induced by the water-sheathing, and by the fully submerged water jet, respectively [9]. However, these studies did not consider cavitation effect in AWJ. Consequently, there has been insufficient knowledge for an improved system design so as to reduce nozzle erosion, while making use of cavitation to increase the material removal rate.

In this study, an experimental system was developed and used to induce cavitation in a fully submerged, high pressure waterjet in slurries. An analysis was then carried out to explore the mechanism of the accelerated surface erosion and the effect of cavitation on the surface erosion.

\section{Experiment}

The schematic diagram of experimental setup is illustrated in Fig. 1. Water was pumped through the system at a pressure of 240MPa using a high intensifier pumping system (Model 5X-60 from Flow). Fig. 2(a) shows the vapour bubble cavitating region and Fig. 2(b) shows the nozzle assembly where an orifice of $0.254 \mathrm{~mm}$ in diameter was used as a waterjet nozzle to generate a waterjet. The nozzle was positioned at the inlet of the mixing chamber, so a fixed stand-off distance of $50 \mathrm{~mm}$ could be maintained. The jet was directed tangentially to the target surface, so the full extend of jet erosion can be studied. The nozzle and the specimen were fully submerged into a slurry solution containing mesh 80 garnet abrasives with the average size of $180 \mu \mathrm{m}$, at a concentration of $90 \%$ by weight. The specimen, slurry and the waterjet were enclosed in a tubular shaped chamber made from perspex to enable observation. Commercial aluminium specimens with the module of elasticity, $E=70 \mathrm{GPa}$, and Poisson ratio, $v=0.35$ were selected because of its high erosion rate and its distinguishable surface characteristics exhibited under different experimental conditions. All the experiments were conducted at a fixed pressure in the following two conditions:

(a) Non-cavitating waterjet in which atmospheric air was introduced inside the mixing chamber by the vacuum action of the high speed jet and expanding as two to three large air bubbles, preventing the formation of vapour bubbles and cavitation; and

(b) Cavitating waterjet in which the mixing chamber was sealed from air; and by this way, the tensile force and vacuum generated by the high speed jet induced nucleation of an infinite number of tiny vapour bubbles, forming a milky cloud in the jet boundary layer. In general, 
MADADNIA, J, SHANMUGAM, D.K., NGUYEN, T. and WANG, J., A study of cavitation induced surface erosion in abrasive waterjet cutting systems. 2008 Advanced Materials Research Vols. 53-54 (2008) pp 357362, online at http://www.scientific.net (c) (2008) Trans Tech Publications, Switzerland

the region which cavitation bubbles collapse on the aluminium specimen, and the speed of waterjet, could be controlled by the pump pressure.

The material removed was determined by weighting of the specimen before and after each experiment. Surface morphology of the specimens was inspected and analysed using a 3D digital microscope (Keyence VHX-100).

\section{Results and Discussion on Material Removal Mechanisms}

Fig. 2 shows the milky cloud representing the cavitation region inside the perspex chamber. It was noticed that the nucleated vapour bubbles gradually disappeared in the cavitation region before reaching to the free surface, as bubbles diffused away from the jet, and as the jet is slowed down by slurries and the specimen. It is believed that the vapour bubbles were in a sub-cooled and metastable condition and their collapse was accompanied with condensation shock and the water hammer effects, resulting in an accelerated surface erosion on the specimen, especially in slurries, as reported in $[10,11]$. It is also noticed that the diameter and the divergent angle of the jet increased when cavitated. The cavitation range determines the effective distance where cavitation may enhance material removal or cause severe wear on the mixing tube in AWJ systems.

Figs. 3(a) and 3(b) illustrate two aluminium specimens exposed for 180 seconds under the testing conditions of (a) and (b) described earlier in Section 2. In Fig. 3(a), the cutting zone is characterised by the features that are often found in erosion by a non-cavitating abrasive waterjet, that is, it contains a cutting wear zone and a deformation wear zone. Material removal within the deformation wear region occurs through a cyclic cutting action and commonly results in a wavy surface texture which serves as the division between the cutting wear and deformation wear regions as reported in [12,13]. A further examination of the surface structure of the sample under condition (a) shows a pit-like surface with shear lips, indicating the high plastic deformation on the surface. This confirms that an extensive plastic deformation has contributed to the high residual strength and rapid degradation of this hardening behavior as the depth of subsurface is increased. In this mode, material is locally work-hardened with continual bombardment of waterjet, and eventually removed by ploughing. The "deformation wear zone” in this cutting exists below the cutting wear zone and is typically identified by waviness or striation patterns caused by severe jet deflections. 
MADADNIA, J, SHANMUGAM, D.K., NGUYEN, T. and WANG, J., A study of cavitation induced surface erosion in abrasive waterjet cutting systems. 2008 Advanced Materials Research Vols. 53-54 (2008) pp 357362, online at http://www.scientific.net (C) (2008) Trans Tech Publications, Switzerland

Fig. 3(b) shows the erosion pattern. In the cutting wear zone, the eroded pattern is associated with a larger bore than that in Fig. 3(a), while outside the cutting wear zone it has a smoother surface polished by the multidirectional impacts and bombardments of the cavitating-induced micro-jets. The weight-loss in the specimens was at least $10 \%$ higher than in case (a) with no cavitation. Fig. 4 shows the sketch of three types of accelerated material removal mechanisms in the cavitating conditions, i.e. suction induced shearing, vortex induced micro-jets, and water hammer. These are analysed below.

\subsection{Suction induced shearing}

Surface morphology of the cut specimens are shown in Figs. 5(a) and (b). It can be realized that the erosion mechanism in the case of Fig. 5(b) is characterized by shearing with little deformation wear. In fact, the dominant type of material removal mechanism is by suction induced shearing due to the material's high ductility. When the high velocity jet was focused near parallel to the surface, the pressure difference between the jet and the slurry in the container produced a shear layer. The pressure difference created by the suction was high enough to infuse (suction) the slurry into the waterjet as shown in Fig. 4. The high speed jet eventually developed into an abrasive waterjet to which the presence of abrasives in the slurry generated shear force that caused the material to be removed by shearing. The surface as observed in Fig. 5(b) possesses fine-scale morphology as compared that in Fig. 5(a); this substantiates that the erosion process involved an extensive mechanical deformation.

\subsection{Vortex induced microjet}

Another feature of the erosion produced by the cavitating waterjet is the pitting on the metal surface outside the effective jet cutting circumference or jet boundary, as shown in Fig. 4. The pitting is combined with highly directional suction induced shearing. It seems that a spiral vortex was generated around the high speed jet submerged inside the still solution. Bubbles were generated around the waterjet and collapsed inside the vortex spiral. When comparing the erosion patterns in Fig. 5(a) (no-cavitation) with that in Fig. 5(b) (with cavitation), it can be seen that the pits are more pronounced in the former case. This observation evidences the formation and collapse of vapour bubbles that results in microjets to erode the metal surfaces.

This type of surface pits are generated when a bubble in the flowing liquid collapses in a location close to the metal surface. To quantify, an average "pit density" of the surface exposed to the microjet induced by bubble collapse was measured and it was found to be around $60 \mathrm{pits} / \mathrm{mm}^{2}$. The 
MADADNIA, J, SHANMUGAM, D.K., NGUYEN, T. and WANG, J., A study of cavitation induced surface erosion in abrasive waterjet cutting systems. 2008 Advanced Materials Research Vols. 53-54 (2008) pp 357-

362, online at http://www.scientific.net (c) (2008) Trans Tech Publications, Switzerland

occurrence of pitting is dependent on the velocity of the microjet and exposure time and strongly attenuated by the waterjet. When the microjet impacts on the material surface, material plastic deformation combined with erosion takes place, and pitting is produced in the material surface. Theoretically, the diameter of the pitting is related to the size of the microjet which, in turn, is proportional to the diameter of the incident bubble.

\subsection{Water hammer}

Water hammer damage was produced on the specimen where bubbles in the liquid collapsed within a very short proximity of the solid surface, and consequently the generated microjets from the bubbles impacted on the surface. It may be explained that the fixed cavity is quasi-static and goes through a cyclic process of formation and collapse. The collapse is due to the production of a reentrant jet in the cavity closure region. The energy released by the collapse of the bubbles during cavitation makes the surface plastic resulting in abrasive-embedment on the surface. Concurrently, small travelling bubbles become entrained within the jet and together collide with the specimen at high impact pressure to create the erosion region. The specific occurrence region of water hammers is shown schematically in Fig. 4.

When observing Figs. 5(a) and (b), pitting is prominent in Fig. 5(b) with larger diametric pits. However, Fig 5(a) also exhibits a substantial amount of pitting with smaller diameter. This may be attributed to the fact that the main material removal mechanism of the waterjet without cavitation is by plastic deformation. When material is removed by plastic deformation, the material tends to chip away causing micro-cracks at the broken chip area. This phenomenon is also common in shearing mode (waterjet with cavitation), however it was not possible to distinguish between the pitting caused by water hammer and micro-cracks caused by chip breaking.

Cavitation by slurry jet involves fluid, erodent particles, and target material. Their properties are therefore important in better understanding the erosion mechanisms. There have been attempts to quantify the erodent trajectories and the fluid properties in high velocity jet [14], but the correlation of such studies to the materials response to erosion still seems remote.

\section{Conclusions}

Material removal by the motion of a cavitation liquid has been found to be predominantly influenced by suction induced shearing. A higher material removal rate can be obtained when cavitation is generated in a submerged waterjet system. The understanding of this work 
MADADNIA, J, SHANMUGAM, D.K., NGUYEN, T. and WANG, J., A study of cavitation induced surface erosion in abrasive waterjet cutting systems. 2008 Advanced Materials Research Vols. 53-54 (2008) pp 357362, online at http://www.scientific.net (c) (2008) Trans Tech Publications, Switzerland

guaranties further studies to use the cavitation phenomenon to increase the material removal rate in abrasive jet processing or to reduce the nozzle wear for this technology.

\section{References}

[1] G.W. Howells, Polymerblasting with Super-Water from 1974 to 1989: a Review, International Journal of Waterjet Technology 1, (1990) 1-16

[2] L. Bayvel and Z. Orzechowski, Liquid atomization, Combustion - An International series, Taylor and Francis, (1993).

[3] J. Bardin, E.A. Eisen, S.R. Woskie, R.R. Monson, T.J. Smith, P. Tolbert, K. Hanmond, and M. Hallock, Mortality studies of machining fluid exposure in the automobile industry: A case-control study of pancreatic cancer, American Journal of Industrial Medicine 32 (1997) 240-247

[4] V.R. Mariani, Effect of viscosity of solid-liquid mixture on pump cavitation Bethlehem, Pa.: Fritz Laboratory, Lehigh University, (1966).

[5] J. Madadnia and I. Owen, Accelerated surface erosion by cavitating particulate-ladenflows, Wear, 165, (1993) 113-116

[6] J. Madadnia and I. Owen I, Erosion in conical diffusers in particulate-laden cavitating flow, Int. Jour of Multi-Phase Flows 21 (1995) 1253-1257.

[7] I. Owen and J. Madadnia Valve Erosion In Particulate-Laden Cavitating Flow, ASME FED- 226 (1995) 59-62.

[8] J. Madadnia,and J. Reizes, Feasibility Studies using cavitation to reduce nozzle erosion in particulate-laden water jet cutting devices, Abrasive Technology - Current Development and Application I, J. Wang et al. (Eds), World Scientific, Sigapore, (1999) 299-305.

[9] G.W. Vickers, F.W.Harrison, R.Houlston, Extending the range of Cavitation cleaning jets, paper J1, $5^{\text {th }}$ International Symposium on Jet Cutting Technology, Honover, Germany, June 1980, 403-412

[10] T. Momma and A. Lichtarowicz, Some experiments on cavitation damage produced by a submerged jet, Proceedings of 2nd ASME-JSME Nuclear Joint Conf. (1993) 877-884.

[11] Z. Sun, X.Q. Kang, and X.H. Wang, Experimental system of cavitation erosion with water-jet, Materials \& Design 26(1) (2005) 59-63.

[12] D. Arola and M. Ramulu, Mechanisms of material removal in abrassive waterjet machining of common aerospace materials, Proceedings of 7th American waterjet conference 1 (1993) 43-64. 
MADADNIA, J, SHANMUGAM, D.K., NGUYEN, T. and WANG, J., A study of cavitation induced surface erosion in abrasive waterjet cutting systems. 2008 Advanced Materials Research Vols. 53-54 (2008) pp 357362, online at http://www.scientific.net (C) (2008) Trans Tech Publications, Switzerland

[13] M. Hashish, A model for abrassive-waterjet (AWJ) machining, Transactions of ASME 111 (1989) 154-162.

[14] H. Liu, J. Wang, N. Kelson and R.J. Brown, A study of abrasive waterjet characteristics by CFD simulation. Journal of Materials processing Technology 153-154 (2004) 488493. 
MADADNIA, J, SHANMUGAM, D.K., NGUYEN, T. and WANG, J., A study of cavitation induced surface erosion in abrasive waterjet cutting systems. 2008 Advanced Materials Research Vols. 53-54 (2008) pp 357362, online at http://www.scientific.net (c) (2008) Trans Tech Publications, Switzerland

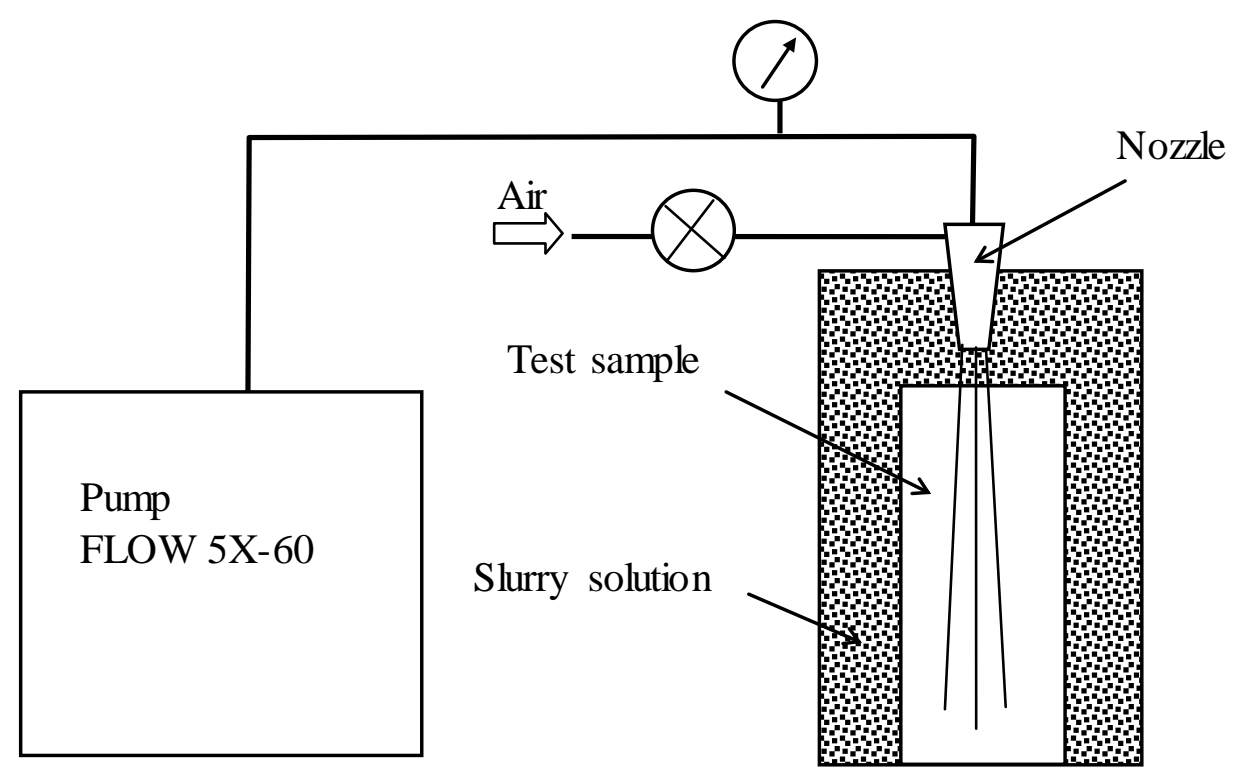

Figure 1. The schematic diagram of the experimental setup. 
MADADNIA, J, SHANMUGAM, D.K., NGUYEN, T. and WANG, J., A study of cavitation induced surface erosion in abrasive waterjet cutting systems. 2008 Advanced Materials Research Vols. 53-54 (2008) pp 357362, online at http://www.scientific.net (C) (2008) Trans Tech Publications, Switzerland

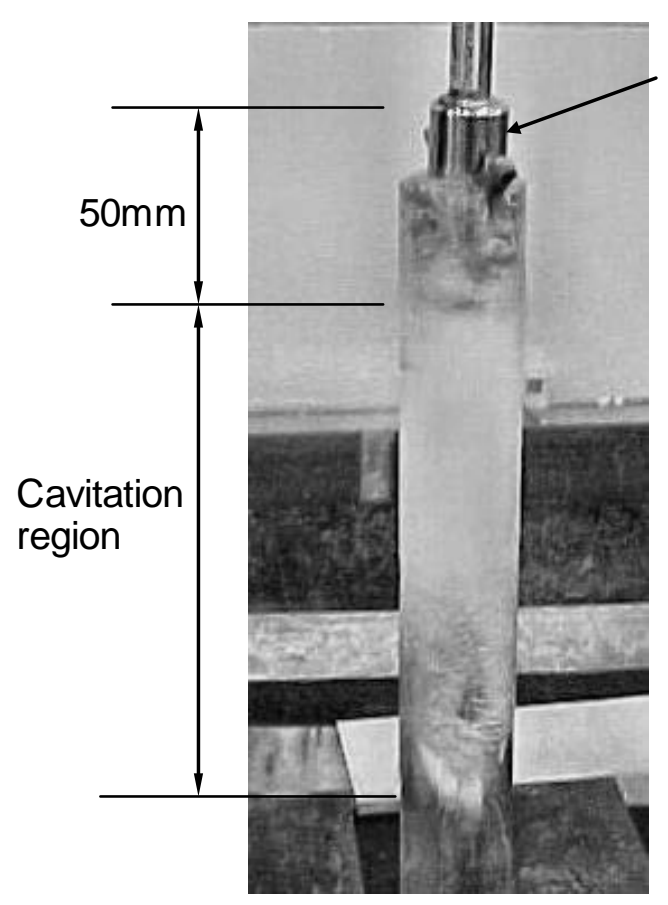

(a)
Mixing

chamber

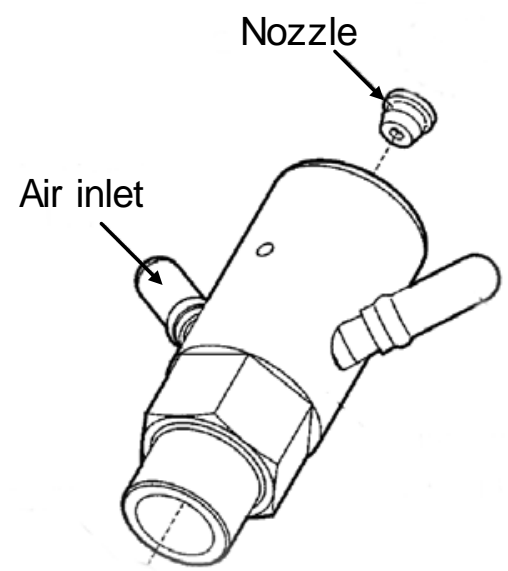

(b)

Figure 2. (a) The vapour bubble region in a high speed cavitating waterjet, and (b) nozzle assembly. 
MADADNIA, J, SHANMUGAM, D.K., NGUYEN, T. and WANG, J., A study of cavitation induced surface erosion in abrasive waterjet cutting systems. 2008 Advanced Materials Research Vols. 53-54 (2008) pp 357362, online at http://www.scientific.net (C) (2008) Trans Tech Publications, Switzerland

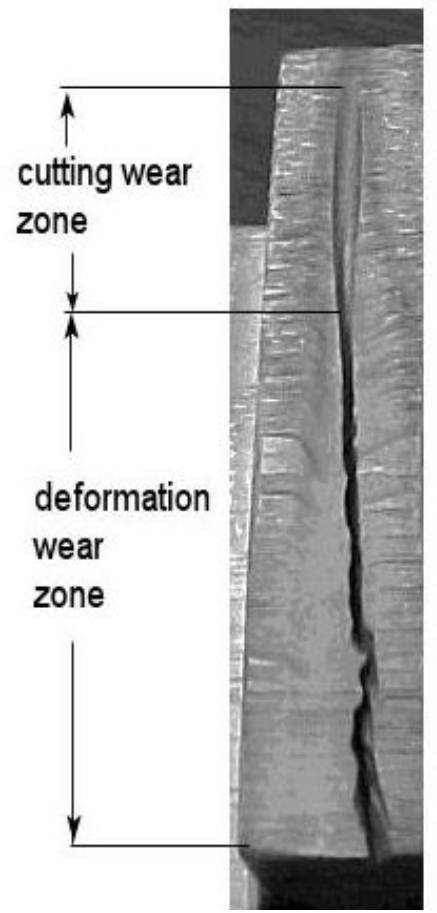

(a)

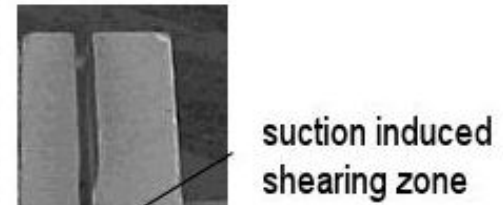

vortex induced

microjet zone

$10 \mathrm{~mm}$

Figure 3. Erosion on $10 \mathrm{~mm} \times 180 \mathrm{~mm}$ aluminium samples after 180 seconds operated at pressure of 240MPa using a jet with diameter of $0.254 \mathrm{~mm}$ : (a) by air-entrained waterjet (no-cavitation) and (b) by vapour-entrained cavitating waterjet. 
MADADNIA, J, SHANMUGAM, D.K., NGUYEN, T. and WANG, J., A study of cavitation induced surface erosion in abrasive waterjet cutting systems. 2008 Advanced Materials Research Vols. 53-54 (2008) pp 357362, online at http://www.scientific.net (c) (2008) Trans Tech Publications, Switzerland

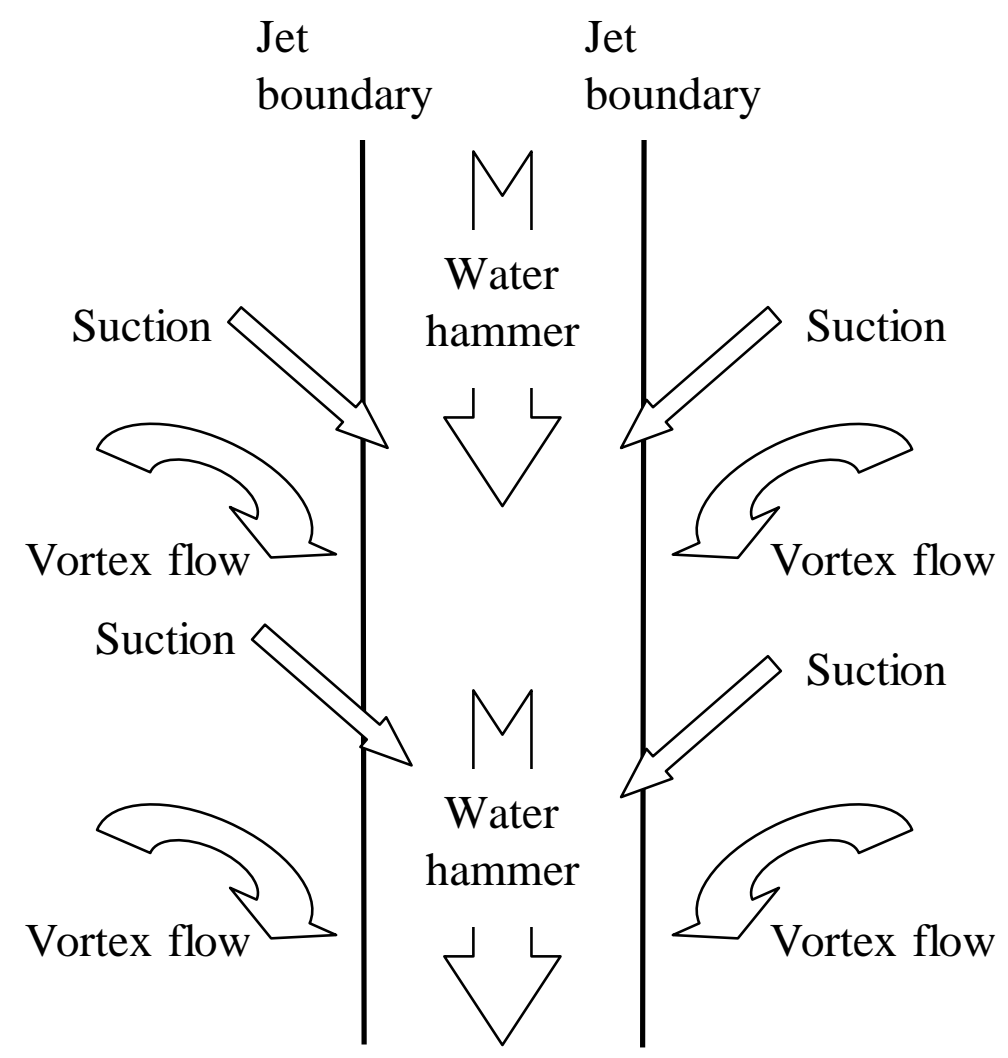

Figure 4: Schematic diagram of material removal mechanism in a cavitating jet showing erosion by suction induced shearing, vortex induced microjet and water hammer. 
MADADNIA, J, SHANMUGAM, D.K., NGUYEN, T. and WANG, J., A study of cavitation induced surface erosion in abrasive waterjet cutting systems. 2008 Advanced Materials Research Vols. 53-54 (2008) pp 357362, online at http://www.scientific.net (C) (2008) Trans Tech Publications, Switzerland

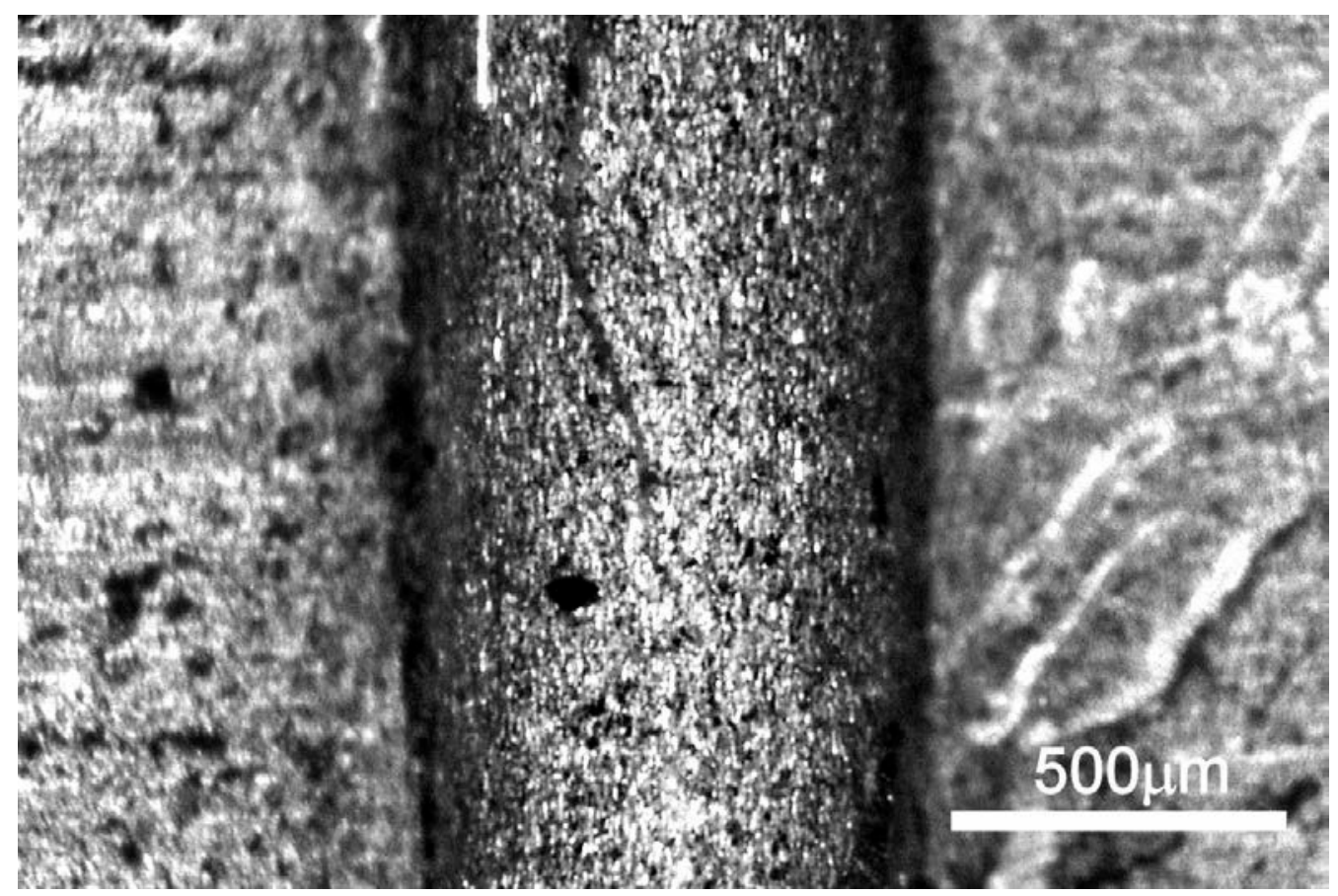

(a)

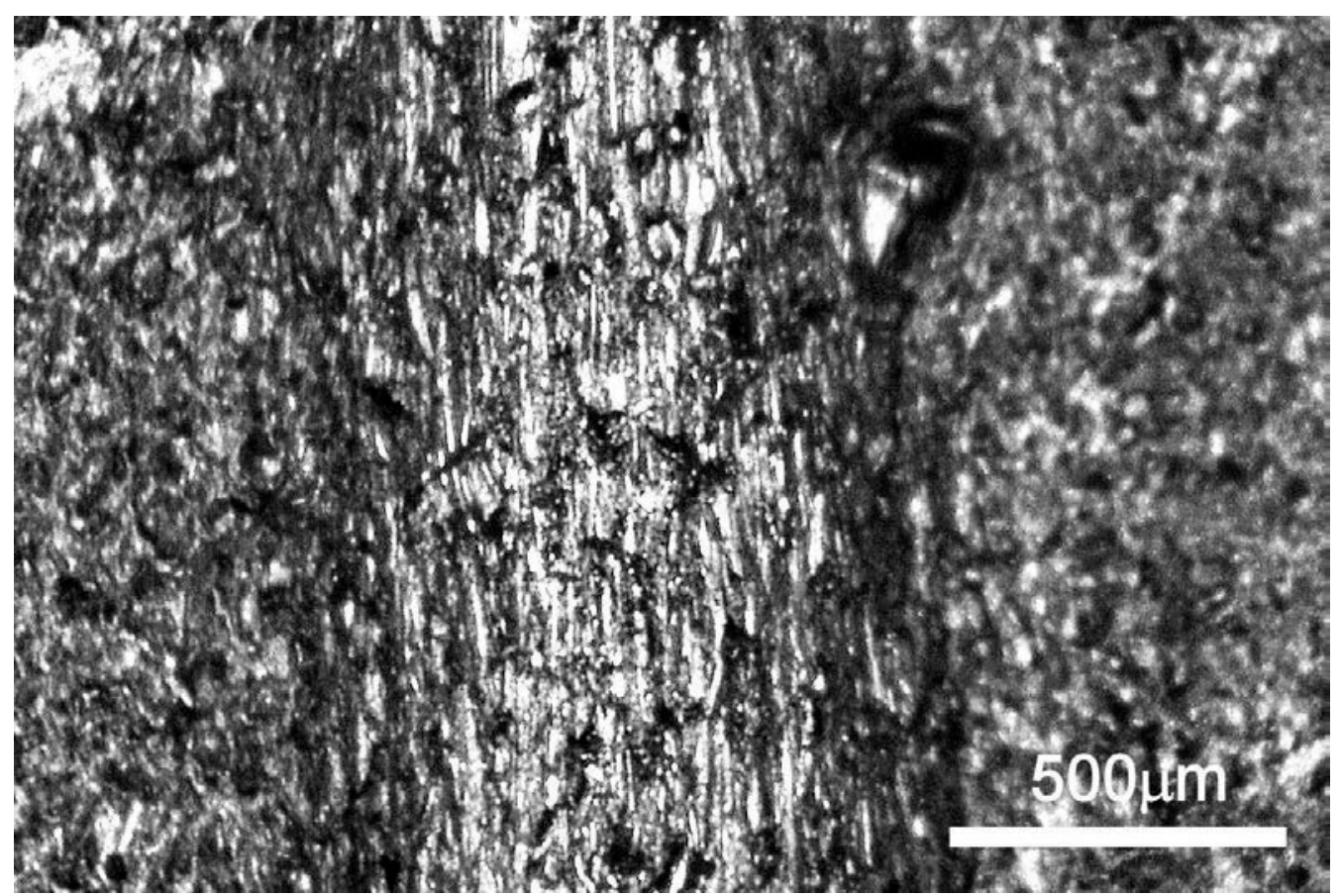

(b)

Figure 5. Surface morphology of aluminium samples eroded by (a) air-entrained waterjet without cavitation, and (b) vapour-entrained cavitating waterjet. Operating conditions are as per in Fig. 3. Noting on shearing mode of material removed within the jet boundary and larger pits outside the boundary in (b) compared to (a). 